\title{
Model for the Prediction of Surface Finish in Ball End Magnetorheological Finishing Process
}

\author{
Saraswathamma. $\mathrm{K}^{\# 1}$, Padmalatha TSRV ${ }^{\# 2}$ \\ ${ }^{\# 1,2}$ Department of Mechanical Engineering, Osmania University, Telangana, India \\ 1saraswathi.ouce@gmail.com \\ 2tsrvpadma@yahoo.co.in
}

\begin{abstract}
Ball end magneto-rheological finishing (Ball end MRF) process possesses the ability of finishing flat and 3D surfaces of magnetic and non-magnetic materials. In the present study, an effort has been made to understand the mechanism of material removal and surface finish in Ball end MRF process on a ferromagnetic (EN 31) workpiece. Magnetic field intensity was simulated in Ansoft Maxwell by varying the gap between workpiece surface and tip of the tool i.e. working gap and magnetizing current. Then magnetic field induced normal force on the abrasive was calculated. Using indentation theory, material removal and percentage change in surface roughness were calculated. The experimental data from literature is used to examine these theoretical results. Theoretically attained results are in close agreement with the experimental results. This demonstrates near closeness of simulated model with the finishing mechanism of Ball end MRF. This surface roughness model further makes the process easier and large investment of time in experimental investigation can be reduced.
\end{abstract}

Keywords-Ball end MRF, EN 31, Magnetic field intensity, modelling, surface roughness.

\section{INTRODUCTION}

Today's advanced engineering industries such as automobile, medical, electronics, and aviation, require high precision finishing methods to tackle the designer's requirements on the components such as, exceptionalmaterial properties, multifaceted 3D components, miniscule features and nano-level finish on complicated geometries etc., [1], [2]. Variousmanufactured products existing in the market require high level finish to augmentthe life and performance by reducing the friction, optical losses and fluid flow resistance. Some products require superior level surface finish such as silicon in semiconductor industries, optics and freeform surface in medical science, micro channels in micro fluidics, moving assembly for instance piston-cylinder and bearings in automobile, and thousands of turbulated cooling holes in a turbine blade[2]. Abrasive-based conventional finishing processes alone are not capable of generating the required surface characteristics to encounter present day demands of nanotechnology. Though in certain situations these methods can be used, but they involve exclusive equipment and sizable labour; finally these processes are not economically viable option.

To overcome the limitations such as high direct labor cost and finishing precision parts of specific geometric features presenting inaccessible areas, advanced fine finishing processes such as Magnetic Abrasive Finishing (MAF), Chemo-Mechanical Finishing (CMP), Abrasive Flow Finishing (AFF), Magnetic Float Polishing (MFP), and Magnetorheological Finishing (MRF) were developed. These processes possess inherent capabilities of finishing various form of geometries with the desired dimensional accuracy and surface finish[3]. To finish the components with tight tolerances and without spoiling the surface topography, precise control of finishing forces is essential. Some of the fine finishing processes such as AFF and CMP are incapable of controlling the abrading forces and hence lack determinism. Many newly developed fine finishing processes such as Magnetic float polishing (MFP), Magnetic abrasive finishing (MAF), and Magnetorheological finishing (MRF) use magnetic field to regulate the abrading forces.

Conventional finishing methods for finishing semiconductor wafers, lenses, and ceramics are labor intensive and difficult to control the forces and moreover these are expensive. The main difficulty in manufacturing high accuracy lenses is that, they are generally made of glass i.e. brittle material, which tends to crack formation during machining. Even a single micro crack can drastically affect the lens's functioning which leads to a situation completely ineffective for the proposed application.

MRF process is a revolutionary precision polishing process and has the ability to finish flats, spheres, and aspheres[4]. This process utilizes stiffened magnetorheological (MR) polishing fluid as a polishing tool. MR polishing fluid is a mixture of micron sized Carbonyl Iron Particles (CIP) and non-magnetic particles i.e. abrasives that are uniformly distributed in carried medium along with additives. MRF is sub-aperture process that is able to finish to an accuracy of $30 \mathrm{~nm}$ peak to valley and surface roughness less than $1 \mathrm{~nm}$ on optical glasses, glass ceramics and hard crystals [4]. Due to restriction on relative movement of finishing medium and workpiece, applications of MR polishing based processesare limited to specific geometries such as concave, 
convex, flat and aspherical shapes only. To overcome these limitations of aforesaid finishing processes, a new precision finishing process i.e. ball end Magnetorheological 'finishing (Ball end MRF) process for Nanofinishing of flat and 3D surfaces of magnetic and non-magnetic materials was developed [5]. In this process a small hemi-spherical ball shaped MR polishing fluid is formed at the tool tip due to variation in magnetic field and this is used to abrade the material from the workpiece. Computer controlled 3 axis motion controller provides movement to the hemi-spherical ball shaped MR polishing fluid. This process finds its potential applications in automotive, aerospace, moulds manufacturing, semiconductor and optics machining etc. [6].

Mori[7] examined the magnetic field, acting forces and provided a fundamental understanding of the process mechanism in MAF. They explained the formation of magnetic abrasive brush from the viewpoint of the brush forming energy. Kim and Choi [8] developed surface roughness model for MAF process to predict finishing time at which existing scratches are to be removed. They developed a model based on micro cutting mechanism and the main assumption is that, surface of the workpiece has uniform profile without statistical distribution having initial surface roughness and abrasives move in lengthwise direction of the scratches. Jha and Jain [9] proposed a model for the CIP chain structures based on microscopic study and possible arrangement of CIP and abrasive with different sizes in the MR polishing fluid composition. Their simulation results shows that, ratio of CIP and abrasive plays crucial role in final surface roughness in MRAFF process. Sidpara and Jain [10], [11] proposed a theoretical model of normal force and tangential force acting on the workpiece in the MR fluid based finishing process and validated it experimentally. They proposed two different theories to model the effect of abrasive particle concentration in the MR polishing fluid. They calculated normal force, tangential force by considering the centrifugal force, Coriolis force respectively.

Based on the literature survey, it is perceived that less attempt has been made towards the modelling and simulation of the Ball end MRF process. Most of the work performed on this process is purely experimental and these studies include the effect of process principle parameters on the material removal and surface roughness. There is no model for representing surface roughness in Ball end MRF process. Hence, the present work is focused on formulation of mathematical model for the surface roughness. If a credible surface roughness model is available, then further improvising the process becomes easier and large investment of time in experimental investigation can be reduced.

\section{BaLl END MRF Process}

Schematic diagram of the Ball end MRF is shown in Fig. 1. The MR polishing tool is placed vertically on a $\mathrm{Z}$-slide, so that tool tip can reach the workpiece surface and required working can be controlled by the Z-axis stepper motor [6]. It alsoincludes a concentric inner core, an electromagnet coil and an outer core. Servomotor is used to control the finishing tool. Workpiece is mounted on a rectangular platform which is formed by X-Y linear movement slides. Three stepper motors are used for controlling the linear motion in the X-Y-Z directions. Computer integrated motion controller is used to accurately regulate the rotational speed of the tool and the three axis motion of the polishing spot. Experiments demonstrated that surface finish of $70 \mathrm{~nm}$ and $102 \mathrm{~nm}$ was achieved on EN31 and copper workpiece respectively [6]. The performance of Ball endMRFmainly depends on the nature of the workpiece material i.e. magnetic and non-magnetic, magnetizing current, working gap, size and concentration of the CIP and abrasives etc.[12] .

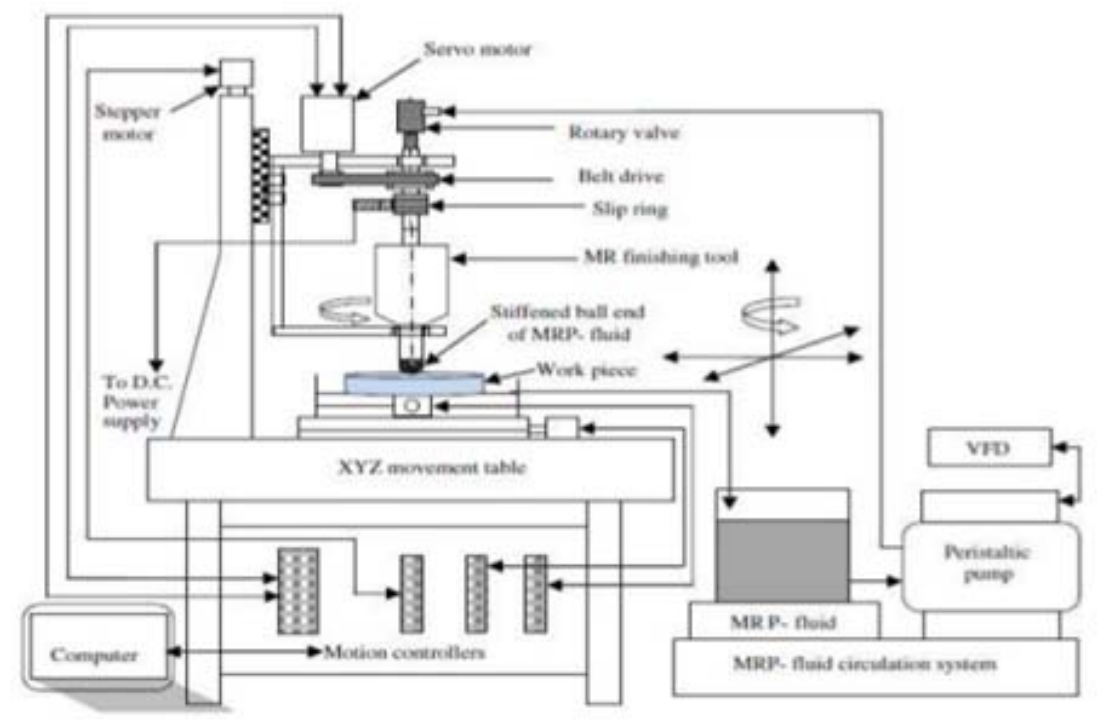

Fig. 1. Schematic of Ball end Magnetorheological finishing [6] 


\section{SIMULATION OF BALL END MRF PROCESS}

Description of physical phenomenon in Ball end MRF process is complex and unpredictable due to non-Newtonian nature of MRP fluid and complex geometry. Hence finite element analysis were carried out to simulate the Magnetic field intensity in the working gap using Ansoft Maxwell. A model similar to Ball endMRF experimental setup is made in the Maxwell as shown in Fig. 2.

The prepared model is analysed by giving different excitations in the range of 2.3-5.7A current and working gap from 0.66 to $2.34 \mathrm{~mm}$. A set of combinations of current and working gap from the reference paper [13] are utilized for determining the Magnetic field intensity. Simulation results representing magnetic field intensity overlay and its vector form are shown in Figs. $3 \& 4$ respectively for a combination of current at 3A and working gap at $1.5 \mathrm{~mm}$. Similarly other combinations of current and working gap are simulated.

Magnetic field intensity for different magnetizing currents are calculated in terms of different working gaps and have been curve fitted as shown in Fig. 5. Fig. 5 represents $3 \mathrm{~A}$ current and different working gaps only. The same process is adopted for remaining.Further, these theoretical values of $\mathrm{B}$ are used to calculate the gradient of $\mathrm{B}$ i.e. $\partial \mathrm{B} / \partial \mathrm{x}$. Mass susceptibility of CIP-CS for different magnetic field densities were calculated from M-B curve of CIP-CS[14]. Typical calculations is shown for 3A current in Eqs. (1) \& (2).

$$
\begin{gathered}
B=0.158 x^{2}-0.837 x+1.365 \text { for cuurent of } 3 A \text { (1) } \\
\frac{\partial B}{\partial x}=0.316 x-0.837(2)
\end{gathered}
$$

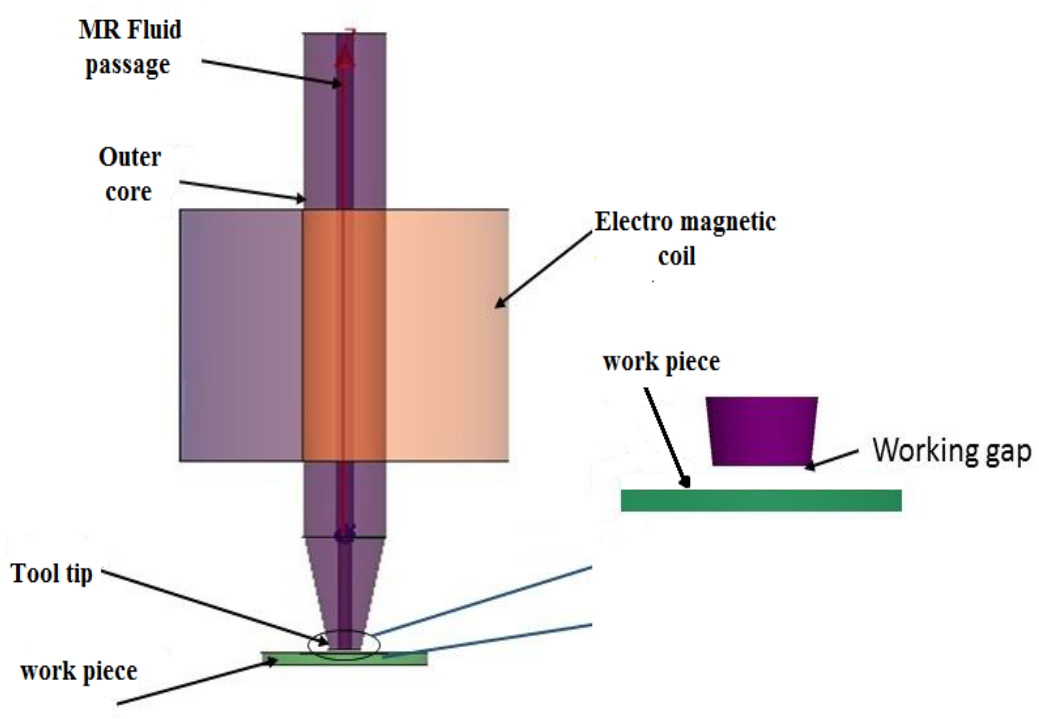

Fig.2. Finite element model of Ball endMRF

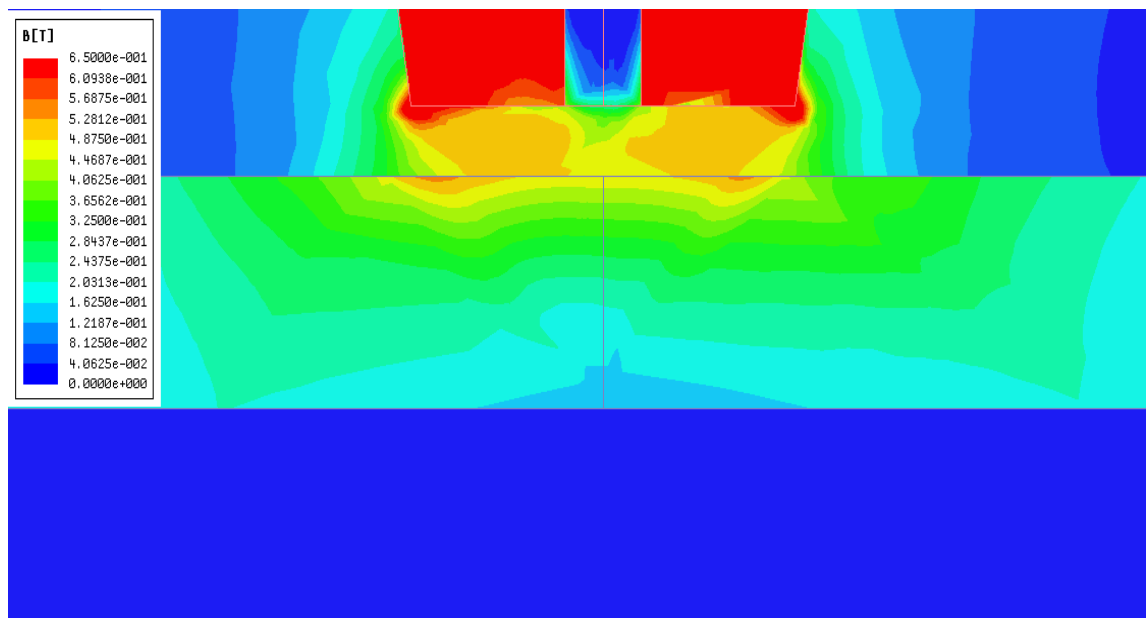

Fig.3.Representation of Magnetic flux field overlay for 3A current and $1.5 \mathrm{~mm}$ working gap 

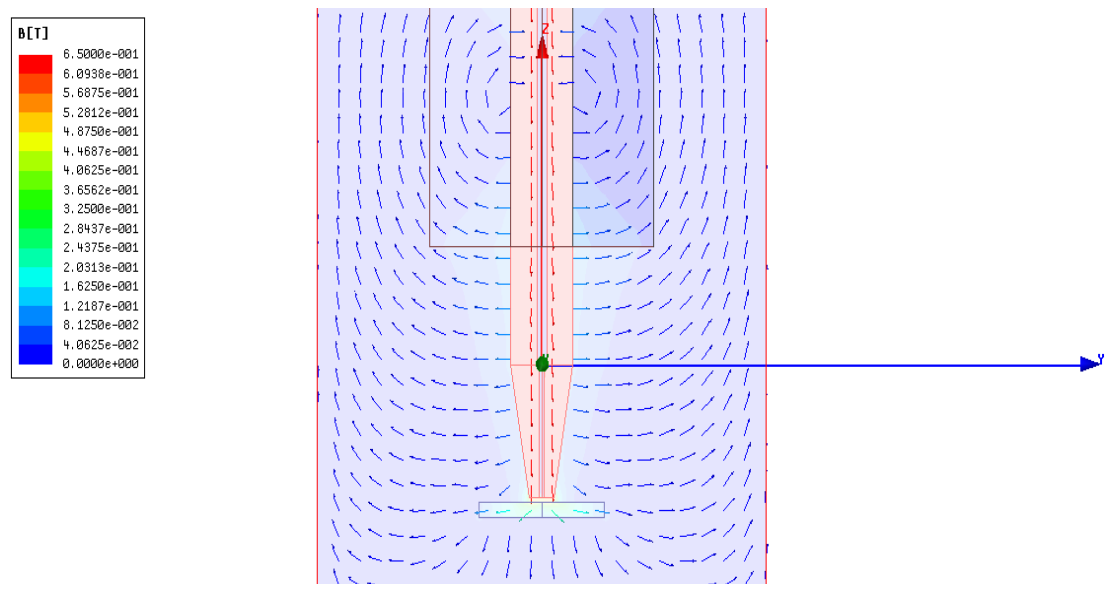

Fig. 4. Representation of $\mathrm{B}-$ vector for $3 \mathrm{~A}$ current and $1.5 \mathrm{~mm}$ gap

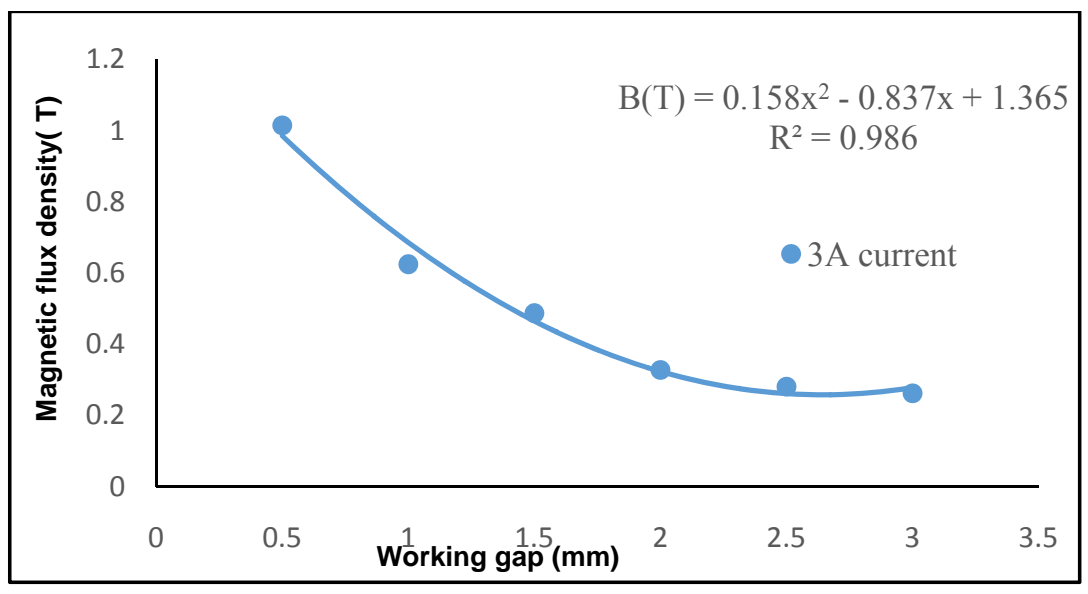

Fig. 5. Magnetic field intensity variation in working gap

\section{A. Calculation of magnetic field induced normal force}

The CIPs in MRP fluid first develop chains between the two electromagnet poles upon the application of magnetic field. The formation of chains is easy to understand because the dipolar energy has its minimum value when two dipoles join together and align in the field direction. These chains then aggregate to develop columnar structure. These columnar structures will form a crystalline structure, which is body centered tetragonal (BCT) structure [15].

Experimentally it has been understood that the outcome surface finish of the workpiece depends on the magnetizing current and working gap. It is required to evaluate the normal force on $\mathrm{SiC}$ abrasive due to surrounding CIPs in the magnetic field to examine the effect of both working gap andmagnetizing current on the surface finish of the workpiece.

The force on a tiny magnetic particle of mass ' $m$ ' in a magnetic field is specified by Eq. (1)[16]

$$
F_{m}=\mu_{0} \chi_{m} \mathrm{H} \nabla H
$$

It is beneficial to substitute field strength with Magnetic field intensity using relation $\mathrm{B}=\mu_{0} \mathrm{H}$ in Eq. (1), which gives Eq. (2)

$$
F_{m}=\left(\mu_{0} \chi_{m} \mathrm{~B} \nabla B\right) / \mu_{0}
$$

where, $\chi_{m}=$ mass susceptibility of the $\mathrm{CIP}(\mathrm{m} 3 / \mathrm{kg}), \mathrm{B}=$ Magnetic field intensity $(\mathrm{T}), \nabla \mathrm{B}=\partial \mathrm{B} / \partial \mathrm{x}, \mu 0=$ Free space permeability $(4 \Pi * 10-7 \mathrm{~Wb} / \mathrm{A}-\mathrm{m}), \rho=$ density of the magnetic particle $=7800 \mathrm{~kg} / \mathrm{m} 3$. Assuming spherical shape of CIP, mass of the CIP in $\mathrm{kg}=\mathrm{m}=\rho v=\rho^{*} 4 / 3$ Пr13. According to the reference [13], radius of CIP, $\mathrm{rl}=$ $9 \mu \mathrm{m}$ and radius of $\mathrm{SiC}$ abrasive, $\mathrm{r} 2=9.5 \mu \mathrm{m}$ was considered. Thus, both ferromagnetic particles (CIP) and abrasive particles are almost of the same size.

\section{B.Active abrasive particles in the MR polishing fluid}

The structure of CIP around abrasive as shown in the Fig. 6 is considered to calculate the normal force on the work-piece by each abrasive particle [15]. While examining the fluid under microscope it has been found that in MRPolishing fluid, abrasives are randomly distributed over the fluid and embedded in between the chains of CIP[9]. In view of these facts and for the simplification in calculation, the following assumptions are made. 
- All the magnetic particles are forming chains.

- Theabrasives which are taking part in material removal are forms a half-BCC structure as shown in Fig.6. This structure consists 4 CIPs at 4 corners and one abrasive particle at the center in contact with the workpiece surface.

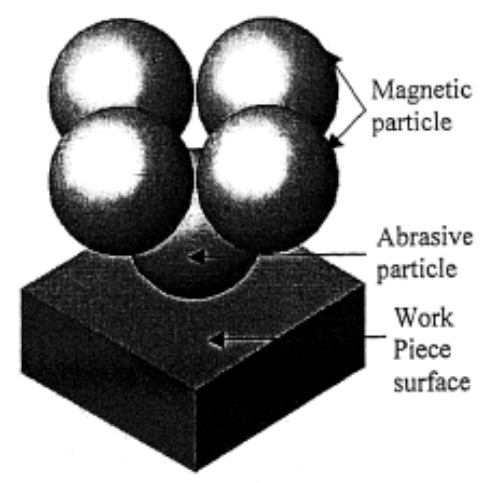

Fig.6. A single half-BCC structure on the work-piece surface [15]

Assuming a unit volume of the working zone $(1 * 1 * 1) \mathrm{mm}^{3}$, number of CIPs $(\mathrm{N})$ per unit volume is given by

$$
\mathrm{N}=\frac{\emptyset_{v}}{\frac{4}{3} \pi \mathrm{r}_{1}^{3}}
$$

where, $\varnothing_{v}=$ Volume concentration of CIPs and $\mathrm{r}_{1}=$ radius of the CIP. Considering that all CIPs are forming vertical chains, the number of chain ends $\left(\mathrm{N}^{*}\right)$ per unit area can be obtained as follows.

$$
\mathrm{N}^{*}\left(\frac{1}{2 r_{1}}\right)=\frac{\emptyset_{\mathrm{v}}}{4 / 3 \Pi \mathrm{r}_{1}^{3}}=\frac{3}{2}\left(\frac{\emptyset_{\mathrm{v}}}{\pi \mathrm{r}_{1}^{2}}\right)
$$

Since only those abrasive particles (active particles) that are trapped in between the magnetic particle chain ends will take part in material removal, hence only these abrasive particles are taken part into consideration. The active (abrasive) particles and chain ends can be chaotically distributed on the surface of the work-piece.

\section{Calculation of normal force acting on SiC abrasive particle}

The chain-structure in the MR polishing fluid near the work piece surface is assumed to be half-body centred cubic. Hence for single active abrasive on the surface of the workpiece surface, there are 4 CIPs surrounding it for a specific fluid concentrationand each CIP exerts force on 4 abrasives as can be seen in Fig. 7. The CIPs experience the magnetic force when magnetic field is applied. This force is transferred to an active abrasive particle due to four surrounding CIPs is equal to F. Considering a single column chain structure, in the first layer active abrasive particle is touching the work piece surface and the force acting is given by the Eq.5.

$$
F_{m}=\left(\mu_{0} \chi_{m} \mathrm{~B} \nabla B\right) / \mu_{0}
$$

Using the above relation, force for combinations of different currents and working gaps is solved using MATLAB program.

\section{SURFACE FINISH MODELING}

For the analysis of material removal and surface finish in Ball end MRF process of EN material, the following assumptions are considered.

- AllCIPs and abrasives are of uniform size and there is a homogeneous distribution of abrasive and CIP in MRP fluid developing a uniform body centered chain structure, which may not be factual in practical case.

- Shape of abrasives and CIPs are approximated to be spherical. Microscopic observation of abrasive particles also illustrates that, they are usually rounded with some irregularities in shape and do not have sharp cutting edges.

- Each abrasive grain consists ofoneeffective cutting edge. If more cutting edges also exists on one grain, then there is noroom for the chips to store in between the cutting edges.

- Every single abrasive grain is supposed to attain the same penetration depth subjected to the applied force for a specified workpiecematerial.

- Whenever grain work piece interference takes place, the mechanism of material removal is presumed by indentation and translation of abrasive grains on workpiece surface. This mode of material removal is assumed to simply the analysis. 


\section{A Material removal model}

The penetration of spherical abrasive grain on workpiece surface takes place due to the normal force acting on it. When the abrasive grain is translated horizontally, the plastically deformed zone beneath the surface will be inclined and give rise to upward flow thus forming a chip which is subsequently sheared from the surface [9].

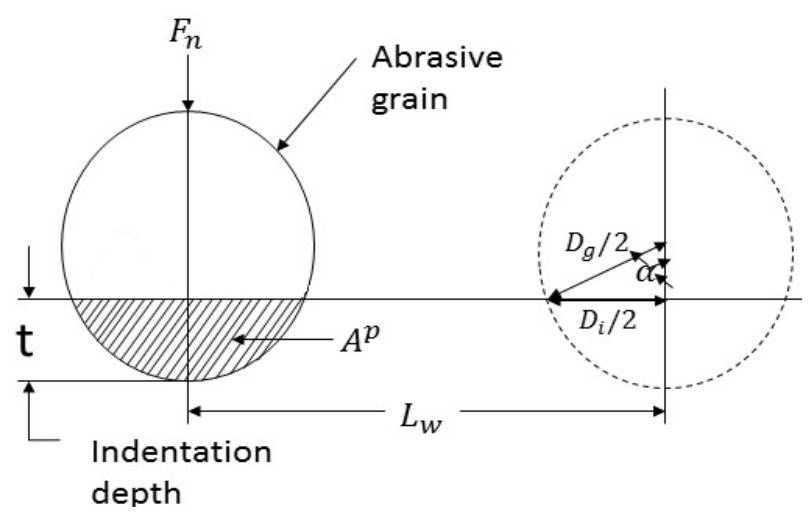

Fig.7. Indentation sketch of $\mathrm{SiC}$ abrasive on the EN31workpiece surface [9]

The Brinell hardness number (BHN) is computed as ratio of load to the indentation surface area and is given by Eq. (6)

$$
B H N=\frac{F}{\left(\frac{\pi}{2}\right) \operatorname{Da}\left(D_{a}-\sqrt{D_{a}^{2}-D_{i}^{2}}\right)^{2}}
$$

where, $\mathrm{F}=$ indentation force on the surface of the workpiece, $\mathrm{D}_{\mathrm{i}}=$ indentation diameter, $\mathrm{D}_{\mathrm{a}}=$ diameter of the abrasive. Using workpiece hardness value in $\mathrm{BHN}$, diameter of the abrasive particle $\left(\mathrm{D}_{\mathrm{a}}\right)$ and indentation force $(\mathrm{F})$, indentation diameter $\left(\mathrm{D}_{\mathrm{i}}\right)$ is computed by simplifying Eq. (6) to the form

$$
D_{i}=\sqrt{D_{a}^{2}-\left(D_{a}-\frac{F}{\frac{\pi}{2} D_{a} B H N}\right)^{2}}
$$

From the Fig. 7 indentation depth $\mathrm{t}$, and indentation diameter are obtained as

$$
\begin{array}{r}
\mathrm{D}_{\mathrm{i}} / 2=\sqrt{\mathrm{t}\left(\mathrm{D}_{\mathrm{a}}-\mathrm{t}\right)} \\
t=\frac{D a}{2}-\frac{1}{2} \sqrt{D_{a}^{2}-D_{i}^{2}}
\end{array}
$$

The cross sectional area $A^{\prime}$ of the indentation groove produced (abrasive grain shaded section in Fig.7) is obtained as

$$
\begin{gathered}
A^{\prime}=\frac{\pi / 4 D_{a}^{2}}{\pi} \alpha-2\left[\frac{1}{2}\left(\frac{D_{i}}{2}\right)\left(D_{a} / 2-t\right)\right] \\
=\frac{D_{a}^{2}}{4} \sin ^{-1} \frac{2 \sqrt{t\left(D_{a}-t\right)}}{D_{a}}-\sqrt{t\left(D_{a}-t\right)}\left(D_{a} /(2-t)\right)
\end{gathered}
$$

Ball end MRF process removes the peaks from the workpiece surface and it is adopted as initial surface profile of the work piece is uniformly distributed with initial surface roughness $\mathrm{Ra}^{0}$ and abrasives move vertical to the direction of the scratches. The initial surface profile is presumed in the form of triangles as shown in Fig. 8. Fromthe total length of the work piece $\left(l_{\mathrm{w}}\right)$, actual length of contact (La) is calculated as(Shown in Fig.8)

$$
L_{a}=\left(\frac{\nabla L g}{\nabla L g+\nabla L s}\right) L_{w}=\left(1-\frac{R a^{i}}{R_{a}^{0}}\right) L_{w}
$$

where, $\mathrm{R}_{\mathrm{a}}{ }^{\mathrm{i}}=$ Surface roughness after $\mathrm{i}^{\text {th }}$ cycle, $\mathrm{l}_{\mathrm{w}}=$ length of the work-piece and is taken as product of feed, rotational speed and time of process as similar to that ofBall end milling ( $\left.f^{*} \mathrm{~N}^{*} \mathrm{~T}\right)$.

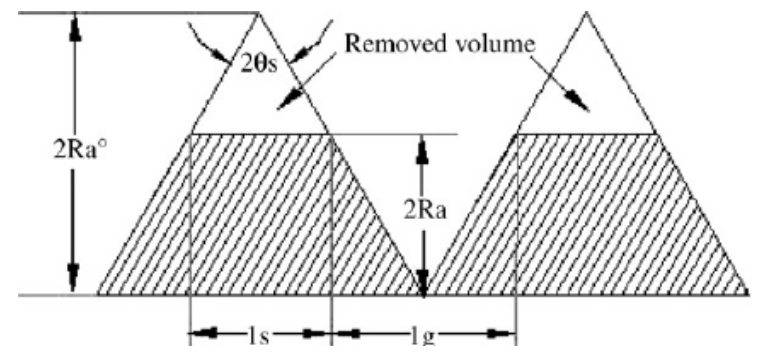

Fig.8. Shape of the scratch machined[9] 
Single abrasive grain volume material removal $\left(\mathrm{V}_{\mathrm{g}}\right)$ is attained using Eq. (10) and (11) as

$$
\mathrm{V}_{\mathrm{g}}=A^{\prime} \mathrm{L}_{\mathrm{a}}=A^{\prime}\left(1-\frac{R a^{i}}{R a 0}\right) l_{w}
$$

From the geometry of the Fig. 8 volumetric material removal (M) in $i^{\text {th }}$ stroke

$$
\begin{aligned}
& \mathrm{M}=\frac{(\mathrm{Ra} 0-\mathrm{Rai})^{2}}{\mathrm{Ra} 0} l_{w}^{2} \\
& \mathrm{R}_{\mathrm{a}}^{\mathrm{i}}=\mathrm{R}_{\mathrm{a}}^{0}-\frac{1}{l_{w}} \sqrt{R_{a}^{0} M}
\end{aligned}
$$

Again, $\mathrm{M}=$ volume of material removed by an abrasive grain $\left(\mathrm{V}_{\mathrm{a}}\right) \times$ total number of active abrasives pitting the workpiece surface. Therefore $\mathrm{M}$ can be written as

$$
\mathrm{M}=A^{\prime} \mathrm{L}_{\mathrm{a}} \mathrm{N}_{\mathrm{a}}=A^{\prime}\left(1-\frac{R_{a}^{i}}{R_{a}^{0}}\right) l_{w} \mathrm{~N}_{\mathrm{a}}
$$

where $\mathrm{N}_{\mathrm{a}}=$ number of active abrasives calculated from Eq. (4)

comparing Eqs. (13) and (14), we get

$$
A^{\prime}\left(1-\frac{R_{a}^{i}}{R_{a}^{0}}\right) l_{w} \mathrm{~N}_{\mathrm{a}}=\frac{(\mathrm{Ra} 0-\mathrm{Rai})^{2}}{\mathrm{Ra} 0} l_{w}^{2}
$$

After simplification, Eq. (15) can be written as

$$
R_{a}^{i}=R_{a}^{0}-A^{\prime} N_{a} / l_{w}(16)
$$

\begin{tabular}{|c|c|c|c|c|c|c|}
\hline \multirow{2}{*}{ S. NO } & \multicolumn{3}{|c|}{ Factors } & \multirow{2}{*}{$\begin{array}{c}\text { \%Change in } \\
\text { Ra } \\
\text { (Experimental) }\end{array}$} & \multirow{2}{*}{$\begin{array}{c}\text { \% Change in } \\
\text { Ra } \\
\text { (Theoretical) }\end{array}$} & \multirow[b]{2}{*}{ \% Error } \\
\hline & $\begin{array}{c}\mathbf{N} \\
(\mathbf{R P M})\end{array}$ & $\begin{array}{l}\text { Current } \\
\text { (A) }\end{array}$ & $\begin{array}{c}\text { Gap } \\
(\mathbf{m m})\end{array}$ & & & \\
\hline 1 & 400 & 5 & 2 & 48 & 40 & 8 \\
\hline 2 & 500 & 2.3 & 1.5 & 40 & 39 & 1 \\
\hline 3 & 500 & 4 & 0.66 & 81 & 57 & 23 \\
\hline 4 & 600 & 5 & 2 & 34 & 47 & -13 \\
\hline 5 & 500 & 4 & 1.5 & 45 & 60 & -15 \\
\hline 6 & 500 & 4 & 1.5 & 47 & 51 & -4 \\
\hline 7 & 500 & 5.7 & 1.5 & 70 & 66 & 3 \\
\hline 8 & 600 & 5 & 1 & 64 & 99 & -36 \\
\hline 9 & 668 & 4 & 1.5 & 37 & 38 & -1 \\
\hline 10 & 400 & 3 & 2 & 37 & 28 & 9 \\
\hline 11 & 500 & 4 & 1.5 & 50 & 36 & 14 \\
\hline 12 & 500 & 4 & 1.5 & 46 & 60 & -14 \\
\hline 13 & 400 & 5 & 1 & 71 & 84 & -13 \\
\hline 14 & 600 & 3 & 1 & 55 & 69 & -14 \\
\hline 15 & 600 & 3 & 2 & 25 & 33 & -8 \\
\hline 16 & 500 & 4 & 1.5 & 49 & 49 & 0 \\
\hline 17 & 400 & 3 & 1 & 60 & 67 & -7 \\
\hline 18 & 332 & 4 & 1.5 & 55 & 34 & 22 \\
\hline 19 & 500 & 4 & 2.34 & 32 & 19 & 13 \\
\hline 20 & 500 & 4 & 1.5 & 45 & 57 & -12 \\
\hline
\end{tabular}

Using the above relation, a program is written in MATLAB to determine roughness values for varied forces and evaluated them with experimental results of Anant et al.[12] and then \% change in roughness is calculated and tabulated in Table 1.

TABLE 1. Percentage variation in surface roughness values $(\%)$ 


\section{RESULTS AND DISCUSSION}

The experimental results of Anant et al. [12] are obtained from regression equation. In order to compare the simulation results with the experimental results, ANOVA was conducted and regression equation is derived from that using Design Expert software.

\section{A. Effect of magnetizing current}

Magnetizing current effect on percentage variation in surface roughness (\% $\Delta \mathrm{Ra})$ presented in Fig. 9. With an increase in the magnetizing current, the percentage variation in surface roughness increases. When current supply to the electromagnetic coil increases, Magnetic field intensity of CIP in the finishing spot of MR polishing fluid increases.Hence, CIP chains hold abrasives more firmly which results an increased finishing action.

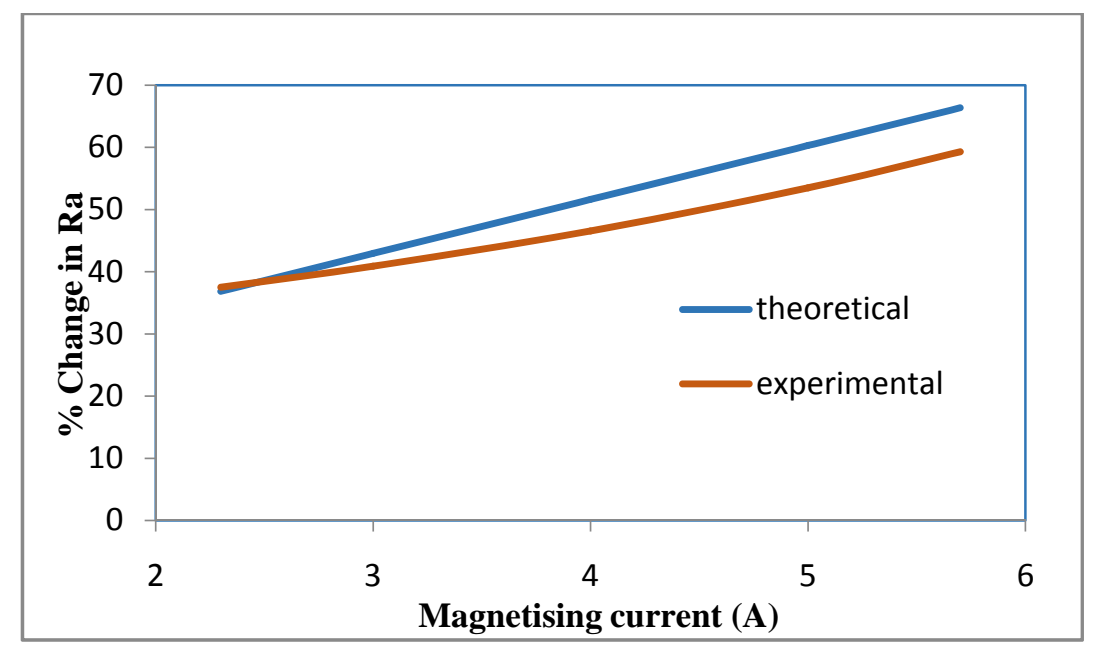

Fig. 9. Effect of Magnetizing current on \%change in Ra

\section{B. Effect of working gap}

Working gap effect on percentage variation in the $R_{a}$ is presented in Fig 10. As the gap between the workpiece and core i.e. working gap, Magnetic field intensity decreases because they are inversely proportional. Hence percentage variation in surface roughness decreases as the gap between the tip surface of the tool core and the work piece surface increases. When working gap increases, Magnetic field intensity of CIP in the finishing spot of MR polishing fluid decreases. Hence, abrasive holding pressure of CIP chains decreases which results decreased in finishing action.

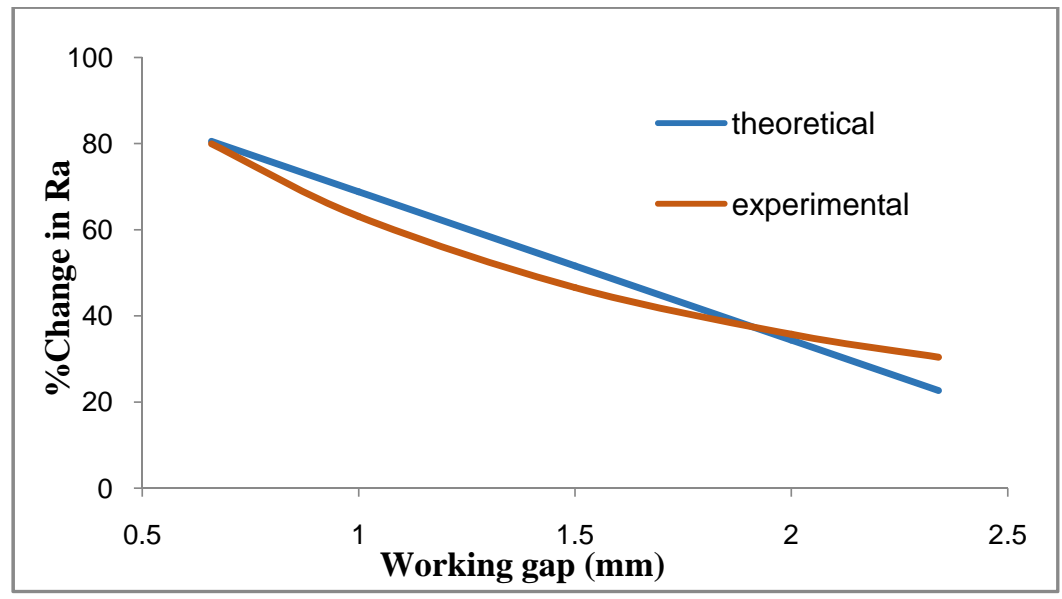

Fig.10. Effect of working gap on \% change in Ra

\section{Effect of rotational speed of tool core}

Effect of rotational speed of the core on percentage variation in surface roughness is presented in Fig. 11. It was observed form the Fig. 11 that, experimental perchance change in surface roughness is decreasing, while simulation percentage variation in surface roughness is increasing. The increase in rotation per min of the central rotating core beyond the given position may reduce the percentage variation in Ra. May be due to the greater centrifugal action on CIP caused by higher working gaps, beyond the exerted interaction force. Since centrifugal force is directly proportional to the square of the core rotational speed, magnetic normal force 
decreases with a rotational speed. Hence, CIPs are not able to grip abrasives strongly during finishing. In simulation, the effect of centrifugal force is not considered, due to this, simulated results are not matched appreciably with the experimental results.

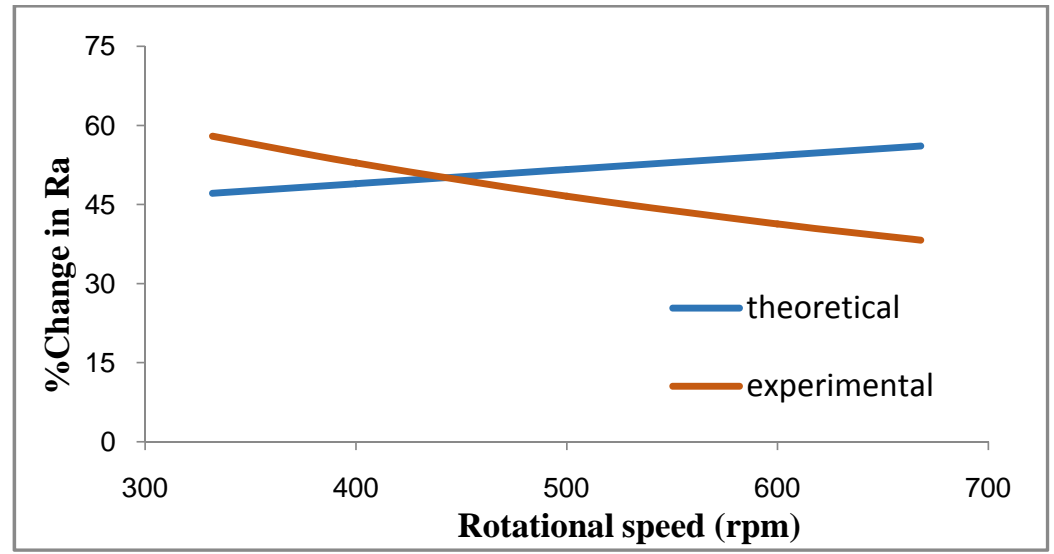

Fig.11.Effect of rotational speed on \%change in Ra

\section{CONCLUSIONS}

Modelling and simulation to predict the surface roughness has been attempted. Magnetic field intensity was simulated in Ball end MRF process using FEM software. On the basis of the results, the following conclusions are drawn.

The magnetic force increases considerably with increase in the Magnetic field intensity and decrease in working gap. Theoretically attained results complements to a greatdegree with the experimental results. This demonstrates nearcloseness of simulated model with the finishing mechanism. On the other side the simulated results on increasing in rotational speed disagree to some extent with experimental results due to nonincorporation of centrifugal force.

\section{REFERENCES}

[1] V. Jain, "Abrasive-based nano-finishing techniques: an overview," Machining Science and Technology, vol. 12, no. 3, pp. 257-294, 2008.

[2] V. K. Jain, A. Sidpara, M. R. Sankar, and M. Das, "Nano-finishing techniques: A review," Proceedings of the Institution of Mechanical Engineers, Part C: Journal of Mechanical Engineering Science, vol. 226, no. 2, pp. 327-346, 2012.

[3] S. Jha and V. K. Jain, "Nanofinishing Techniques," in Micromanufacturing and Nanotechnology: Springer Berlin Heidelberg, 2006, pp. 171-195.

[4] W. Kordonski and D. Golini, "Progress update in magnetorheological finishing," International Journal of Modern Physics B, vol. 13, no. 14 n16, pp. 2205-2212, 1999.

[5] A. Kumar Singh, S. Jha, and P. M. Pandey, "Design and development of nanofinishing process for 3D surfaces using ball end MR finishing tool," International Journal of Machine Tools and Manufacture, vol. 51, no. 2, pp. 142-151, $2 / / 2011$.

[6] A. K. Singh, S. Jha, and P. M. Pandey, "Magnetorheological Ball End Finishing Process," Materials and Manufacturing Processes, vol. 27, no. 4, pp. 389-394, 2012/04/01 2011.

[7] T. Mori, K. Hirota, and Y. Kawashima, "Clarification of magnetic abrasive finishing mechanism," Journal of Materials Processing Technology, vol. 143, pp. 682-686, 2003.

[8] J.-D. Kim and M.-S. Choi, "Simulation for the prediction of surface-accuracy in magnetic abrasive machining," Journal of materials processing technology, vol. 53, no. 3, pp. 630-642, 1995.

[9] S. Jha and V. K. Jain, "Modeling and simulation of surface roughness in magnetorheological abrasive flow finishing (MRAFF) process," Wear, vol. 261, no. 7-8, pp. 856-866, 2006.

[10] A. Sidpara and V. K. Jain, "Analysis of forces on the freeform surface in magnetorheological fluid based finishing process," International Journal of Machine Tools and Manufacture, vol. 69, pp. 1-10, 2013.

[11] A. Sidpara and V. K. Jain, "Theoretical analysis of forces in magnetorheological fluid based finishing process," International Journal of Mechanical Sciences, vol. 56, no. 1, pp. 50-59, 2012.

[12] A. K. Singh, S. Jha, and P. M. Pandey, "Parametric analysis of an improved ball end magnetorheological finishing process," Proceedings of the Institution of Mechanical Engineers, Part B: Journal of Engineering Manufacture, vol. 226, no. 9, pp. 1550-1563, 2012.

[13] A. K. Singh, S. Jha, and P. M. Pandey, "Parametric analysis of an improved ball end magnetorheological finishing process," Proceedings of the Institution of Mechanical Engineers, Part B: Journal of Engineering Manufacture, p. $0954405412453805,2012$.

[14] M. Das, V. Jain, and P. Ghoshdastidar, "Analysis of magnetorheological abrasive flow finishing (MRAFF) process," The International Journal of Advanced Manufacturing Technology, vol. 38, no. 5-6, pp. 613-621, 2008.

[15] M. Das, V. K. Jain, and P. S. Ghoshdastidar, "Analysis of magnetorheological abrasive flow finishing (MRAFF) process," International Journal of Advanced Manufacturing Technology, vol. 38, no. 5-6, pp. 613-621, 2008.

[16] A. W. Stradling, "The physics of open-gradient dry magnetic separation," International Journal of Mineral Processing, vol. 39, no. 1-2, pp. 1-18, 1993. 


\section{AUTHOR PROFILE}

Dr.K.Saraswathamma (Assistant Professor) received her B.E. from Andhra University in 1998 and Ph.D. in 2015 from IIT Delhi. After post-graduation at Osmania University in 2001, she joined in the Department of Mechanical Engineering, College of Engineering, Osmania University in 2001. Her research interests are in the areas of Micro and Nano machining, conventional and Un-conventional machining, application of finite element technique, etc. She has published nearly 6 papers in peer reviewed international journals in addition to 12 presentations in national and international conferences.

Ms.TSRV Padmalatha (Assistant Professor) received her B.Tech from JNTU in 2000, M.Tech. in 2006 from SV University and pursuing PhD at IIT Madras. She joined in the Department of Mechanical Engineering, College of Engineering, Osmania University in 2007. Her research interests are in the areas of Metal forming, conventional and Un-conventional machining, etc. 